\title{
Chronic Energy Deficiency and Associated Factors among Lactating Mothers (15- 49 years old) in Offa Woreda, Wolayita Zone, SNNPRs, Ethiopia
}

\author{
Berhanu Wodajo Julla \\ Abebe Haile ${ }^{2}$ \\ Girma Ayana ${ }^{3}$ \\ Solomon Eshetu ${ }^{4}$ \\ Desalegn Kuche ${ }^{5}$ \\ Tsehai Asefa ${ }^{6}$
}

${ }_{3,4,5,6}$ Ethiopian Public Health Institute, Ethiopia Addis Ababa, Ethiopia

Email:BerhanuW99@gmail.com

Email:girmayayana@yahoo.com

Email:solomoneshetu13@yahoo.com

${ }^{5}$ Email:desukuche@gmail.com

${ }^{6}$ Email:Tsehaiasefa@yahoo.com

'Addis Ababa University, College Development Studies,Centre for Food Security Studies, Addis Ababa, Ethiopia

2Email:abebe.haile@aau.edu.et

\begin{abstract}
The lactating mothers in developing countries like Ethiopia are the first targeted and nutritionally vulnerable groups to under nutrition due to different socio demographic factors which influence the health and well being of mothers and children. During lactation periods, mothers need more nutritious food than the rests of the household members. The aim of this study was to determine the nutritional status, associated factors and dietary diversity of lactating mothers 15-49 years old in Offa woreda. A crosssectional community based study was carried out to determine the Chronic Energy deficiency of lactating mothers in the studied woreda. The lactating mothers were randomly selected from four rural and two urban kebeles. A total of 422 households were visited and the required data were collected as scheduled in all selected kebeles. The prevalence of under nutrition among the lactating mothers in the studied area was determined using BMI by Anthropometric measurements and the blood sample test for hemoglobin (Hb) concentration was done using Haemocue methods. The collected data were analyzed using Stata version 14.2 software. Among the participated lactating mothers the values $15.8 \%, 74.2 \%, 8.3 \%$ and $1.7 \%$ were obtained for under weight, normal, over weight and obese in their BMI $(\mathrm{kg} / \mathrm{m} 2)$, respectively. The studied participants of $15.8 \%$ were in chronic energy deficiency according to WHO guidelines. The lactating mothers' percentage value $36.4 \%, 59.3 \%, 4.3 \%$ were in age group of $15-24,25-34$ and $35-49$ years, respectively. The blood test showed that among the lactating mothers $11.4 \%$ were found anemic which had got hemoglobin level less than normal level $(12 \mathrm{~g} / \mathrm{dl})$. The daily dietary diversity intake per day of lactating mothers was classified as high (>5 DDS) $1.2 \%$, medium (4-5 DDS) $50.7 \%$ and low (<3 DDS) $47.4 \%$ among the nine food groups recommended for women dietary diversity score (WDDS). The independent variables: education, family size, marital status and occupation were significantly associated with nutritional status of the lactating mothers at $\mathrm{P}<0.05$. In conclusion the majority of the lactating women in the study area had normal BMI. The researchers would like to recommend that the government and development agencies should focus on the risk factors identified, to ensure better health and nutrition for lactating mothers in this woreda.
\end{abstract}

Keywords: Nutritional status, Lactating mothers, Dietary diversity, Offa Woreda, BMI.

Citation | Berhanu Wodajo Julla; Abebe Haile; Girma Ayana; Citation Berhanu Wodajo Julla; Abebe Haile; Girma Ayana;
Solomon Eshetu; Desalegn Kuche; Tsehai Asefa (2018). Chronic Energy Deficiency and Associated Factors among Lactating Mothers (15-49 years old) in Offa Woreda, Wolayita Zone, SNNPRs, Ethiopia. World Scientific Research, 5(1): 13-23. History:

Received: 6 November 2017

Revised: 18 December 9017

Revised: 18 Decher 2017

Accepted: 28 December 2017

Published: 10 January 2018

Licensed: This work is licensed under a Creative Commons Attribution 3.0 License (cc)

Publisher:Asian Online Journal Publishing Group Contribution/Acknowledgement: First of all I would like to thank the Almighty
God who is always beside me during my happiness and trouble time. I am very thankful God who is always beside me during my happiness and trouble time. I am very thankful to Addis Ababa Unive sincere gratitude goes to Dr. Abebe Haile, who advised me well while this thesis was prepared from beginning to end. I am very thankful to the Center for Food Security Studies for good administration service offered for any raised issues. I had a great support and encor and encouragements from all families while I was learning and conducting this Research I Would like to say may God bless them. The Regronal Health, Wolayita Zone Health and respective offices. Lastly, I m greatly, indebted to the lactating mothers in Offa Woreda, respective offices. Lastly, I am greatly, indebted to the lactating mothers in Offa Woreda, Wolayita zone, SNNPRs, for their full willingness in responding the questionnaires as great. In general, without the support of EPHI, I can say that this Research would not great. In general, withon

Funding: This study received no specific financial support.

Competing Interests: The authors declare that they have no conflict of interests.

Transparency: The authors confirm that the manuscript is an honest, accurate, and transparent account of the study was reported; that no vital features of the study have been omitted; and that any discrepancies from the study as planned have been explained.

Ethical: This study follows all ethical practices during writing. 


\section{Contents}

1. Introduction

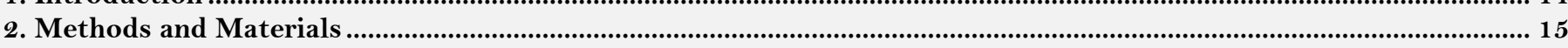

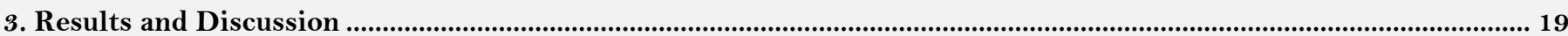

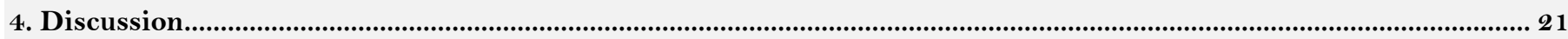

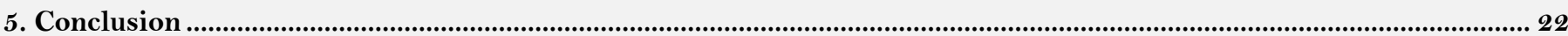

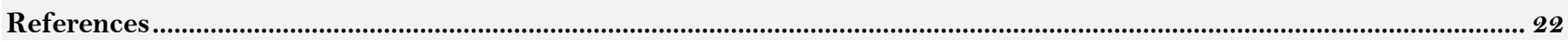

\section{Introduction}

Low dietary diversity and its consequence of under nutrition is a problem of millions of people in the developing world. Low dietary diversity at individual, household and community levels, or a combination of these, may contribute to poor nutrition and health status. In particular, under nutrition among women is likely to have a major impact on their own health and their children. Maternal under nutrition, including chronic energy and micronutrient deficiencies, account for $11 \%$ of the global burden of disease [1]. So many studies had been conducted on maternal nutritional problems in various countries and the outcome showed that maternal under nutrition ranges from $10-19 \%$ in most developing countries. This is usually a result of inadequate dietary intake, occupation, family size, education, food insecurity at household level and other social factors. Maternal and child in low and middle income countries encompasses both under and over nutrition [2]. Lactation places high demands on maternal stores of energy and protein than in any other stage of a woman's reproductive life. To support lactation and maintain maternal reserves, most mothers in developing countries will need to eat about 500 additional kilocalories or an increase of 20 to 25 percent over the usual daily intake.

About 35\% Ethiopian population is chronically undernourished [3]. Twenty seven percent were women aged $15-49$ who had BMI $<18.5 \mathrm{~kg} / \mathrm{m}^{2}$ which remained relatively constant since 2005 as it was reported by CSA [4]. EDHS 2011 report showed that the period 2000-2005 in which significant improvement showed in reduction of under nutrition in Ethiopia, however, it remained constant after the periods of 2005. In year 2000 survey, age marital status, religion, occupation, wealth index were found to significantly affect women's nutritional status. Whereas, in 2005 religion of residence was no longer factor influencing women's under nutrition by EDHS (Ethiopia Demographic and Health Survey) [5]. In EDHS (Ethiopia Demographic and Health Survey) [6] report, socio-demographic characteristics, maternal health care and other household family issues were indicated. At national level, $97 \%$, 56.5\% of urban and rural respectively used to get drinking water from improved source. With regarding to education, $47.86 \%, 35 \%, 11.6 \%, 5.6 \%$ had no education, primary, secondary and above, respectively. The maternal health care was assessed as $63 \%$ and $27 \%$ urban and rural, respectively had antenatal care (ANC) $\geq 4$ times from skilled provider. The ANC coverage in SNNPR was $69.3 \%$ and postnatal care (PNC) within 48 hours check up was $16.9 \%$. The prevalence of anemia in this region was $22.6 \%$ [6].

The study conducted in Nekemte referral Hospital showed, among the participants $20 \%$ of lactating mothers had BMI less than $18.5 \mathrm{~kg} / \mathrm{m}^{2}[7]$. The similar, study conducted in west Showa, Ambo district on household food insecurity and factors associated with under nutrition among lactating mothers in rural Ambo result showed that $21.5 \%$ were nutritionally under weight or their BMI less than $18.5 \mathrm{~kg} / \mathrm{m}^{2}[8]$. Proper food and good nutrition are essential for survival, physical growth, mental development, performance and productivity, health and a well being of all living things WFP [9]. According to EHNRI (Ethiopian Health and Nutrition Institute) [10] nutrition baseline survey reports among non-pregnant women, women with no education were more likely to be underweight $(31 \%)$ where educated $(17 \%)$. The survey of EDHS (Ethiopia Demographic and Health Survey) [6] result showed that the percentages of educated women at different education levels were, women not educated $(47.8 \%)$, primary $(35.0 \%)$, secondary $(11.6 \%)$ and more than secondary $(5.6 \%)$.

Age of lactating mother, place of residence, employment and marital status were found to be significantly associated with nutritional status of women. The prevalence of under-nourished women was also higher among older women [11]. Moreover, women in rural areas were twice more likely to be under-nourished than those in urban areas. According to WHO [12] report, low body mass index $\left(<18 \mathrm{~kg} / \mathrm{m}^{2}\right)$ or short stature height $(<145 \mathrm{~cm})$ are common in women in low-income countries, with the highest rate in southern and south-eastern Asia, followed by sub-Saharan Africa, with "critical" rates ( > 40\%) in Eritrea and Bangladesh, and a "serious" (20 to 39\%) prevalence in Cambodia, Chad, Ethiopia, Indian, Madagascar, Mali, Nepal and Yemen; most other countries have a prevalence chronic energy deficiency between 10 to $19 \%$. More than $10 \%$ of women are shorter than $145 \mathrm{~cm}$ in Bangladesh, India, Nepal and in Latin America and Caribbean countries. An estimated 663 million people worldwide do not have access to an improved drinking-water source [12]. About 2.4 billion people or one third of the world's population, lack access to an improved sanitation facility and $13 \%$ practice open defecation. Among the world's regions, sub-Saharan Africa and South Asia continue to have the lowest sanitation coverage [12]. As the study from SriLanka showed the availability of adequate sanitation facilities, such as safe drinking water and unshared sanitary toilet facilities were important determinants of women nutritional status [12]. In rural areas of Burkina Faso the BMI was significantly associated with the hygienic level of the household and the care for women [13]. Unlike household access to drinking-water and sanitation, no global mechanism exists to monitor hand washing practices in homes and communities.

According to EDHS (Ethiopia Demographic and Health Survey) [6] report, a large proportion of maternal and neonatal occur during the first 48 hours after delivery. Thus, prompt postnatal care (PNC) for both the mother and the child is important to treat any complications arising from the delivery, as well as to provide the mother with important information on how to care for herself and her child. Safe motherhood programs recommended that all women receive a check of their health within 2 days after delivery. Dietary diversity represents the number of different foods or food groups consumed over a reference period-usually 24-hour recall which is designed to capture the micronutrient adequacy of food groups consumed by the individual $[14,15]$. Cross sectional Research exploring the wealth and under nourishment among two impoverished nations, Burkina Faso and Cong Democratic Republic had been conducted to evaluate the under nourished level between the rich and poor of women in two nations. The reported results showed that $19.4 \%$ and $18.4 \%$ the poor against $7.2 \%$ and $9.7 \%$ rich 
women in Burkina Faso and Democratic Republic Congo made assessments, respectively. Besides to this older women were more prevalence of under nourish than younger. According the Burkina Faso and Congo research undernourished women was common among the poor and with no formal education [7]. The problem of most developing countries in the world was not identifying the impotence of micro nutrient intake and dietary diversity. According to Food and Agriculture Organization [16] report, more than 2 billion people were in micro nutrient deficiency which is referred to as hidden hunger."

An estimated 663 million people worldwide do not have access to an improved drinking-water source [12] and an estimated 1.9 billion people rely on drinking-water that is fecal contaminated [17]. Improved water sources that are not operated or maintained properly may deliver water that is microbiologically contaminated that of open defecation. Among the world's regions, sub-Saharan Africa and South Asia continue to have the lowest sanitation coverage [12].

Under nourishment is a worldwide problem of lactating women living in developing countries. Lactating women in south East Asia, sub- Saharan and Africa are highly vulnerable to chronic energy deficiency. As a result of this, the lactating women are exposed to some non- infectious disease caused by the low deficiencies of dietary diversity food. The Research conducted in Pakistan and Nepal showed, 15.1 and $27 \%$ of lactating mothers were in chronic energy deficiency respectively [18].

Evidence from urban slums of North-east district of Delhi, India (2011) showed that the among 160 lactating mothers, after deliverance of three months, $16 \%$ of respondents had a BMI of less than $18.5 \mathrm{~kg} / \mathrm{m}^{2}[19]$. However, the study conducted in Alemtidad area around Khartoum was the inverse result obtained in other parts of developing countries in which no underweight was observed. According to the result, the problem of lactating mothers in Alemtidad was the problem of overweight and obesity. Among the participants only 35.6\% had normal BMI and the rest were observed as overweight and obese. The Global economic crisis, commodity price hikes and climate change have worsened the position of the poorest and most vulnerable people. Thus the crisis compromising the diet and health up to $80 \%$ of population [20]. According to this statement the future generation's nutritional status, especially the developing countries is in threatening condition. In Ethiopia, one of every four or $26 \%$ women of reproductive age were undernourished that is twice the sub-Saharan average of $13.3 \%$ [21]. Comparing the nutritional status of Ethiopian women, with that of women in 29 other sub-Saharan African countries, based on the DHS conducted between 1998 and 2008, the prevalence of under nutrition in Ethiopia is higher when compared with other developing countries. Ethiopia has decreased from $75 \%$ to $35 \%$ over two decades, even though, these positive advances shown in Ethiopia, one in three people live below the poverty line [22, 23].

The food insecurity which contributes to under nutrition of lactating mothers was studied to identify the contribution of food insecurity to under nutrition both on mothers and children. According to the research output women from food insecure households had a lower energy intake than those from food secure households [24]. Food insecurity has a wide range of negative health impacts beyond under nutrition. In Southern Nations, Nationalities and Peoples' Region (SNNPR) so many lactating mothers were suffering from food insecurity. According to Almaz [25] 67.8\% total households of Wolayita zone were under food insecure. Moreover, there was a knowledge gap in utilizing necessary health care as studied around Gonder 'Zuria' and due to this knowledge limit only $66.83 \%$ had utilized ANC service [26].

Offa is one of the densely populated woreda in Wolayita zone and supposed with low dietary diversity intake by lactating mothers. The nutritional status, dietary diversity and the associated factors for chronic energy deficiency were assessed in detail both in selected rural and urban kebeles. There was a gap of information about the prevalence of under nutrition in study area (Offa woreda) and due to this, the high percentage prevalence under nutrition was considered for this woreda to identify the chronic energy deficiency of lactating mothers of 15-49 years in the study woreda.

Thus, the objective of this Research is to determine the nutritional status, dietary diversity and associated factors among the lactating mothers in Offa woreda, Wolayita Zone, SNNPR, Ethiopia having infant/child less than 24 months and breast feeding.

\section{Methods and Materials}

\subsection{Description of Study Area and Duration}

This study was conducted from March 8 to April 13, 2017 in six kebeles, Offa woreda. The selected study area was one of the wolayita zone woreda, SNNPR and the research was conducted to estimate the nutritional status of lactating mothers 15-49 years. Gesuba is the capital town of Offa woreda which is $29 \mathrm{~km}$ from Wolayita Sodo and 414 Km from Addis Ababa capital city of Ethiopia. The woreda is bounded with Kindo Koysha woreda in the north, Gamo Gofa Zone in the south, Humbo and Sodo Zuriya in the East and Kindo Didaye Woreda in the west. Based on National Census the projected total population of the woreda in 2009 E.C was 132,054, out of which, 65,765 and 66,291 were male and female, respectively. There are 21 rural and 2 urban kebeles in the woreda. The total area of the woreda is 38,557 hectare. The cultivated proportional land according to the woreda agriculture Office information was 22,912 hectare or (59.4\%). According to CSA [4] the woreda is situated at 6 $37^{\circ}$ '07.63"N-6 $50^{\circ} 07.10^{\prime \prime N}$, latitude and Longitude: 37\%2418.06”E-37'89'13.27'E and altitude: ranges $1200-2800$ meter above sea level. Geo-ecology of Offa woreda: Kola, Weynadega and Dega (22, 62 and 16\%) respectively The woreda's annual average rainfall, $800-1400 \mathrm{~mm}$ and annual average temperature, $14{ }^{\circ} \mathrm{C}-34^{\circ} \mathrm{C}$ (Offa woreda Agriculture Office).

\subsection{Major Crops and Animals}

The major crops in Offa woreda are: maize, teff, sorghum, wheat, barley, bean, pea, peanuts and chickpea. Different roots and tuber crops like enset, sweet potato, cassava, taro, and white yam were commonly cultivated. There are also fruits and vegetables; mainly mango, banana, avocado, papaya, sugar cane, tomato, onion, cabbage and green paper are common. The animals reared in the study woreda are: Cattle, sheep, goat, chicken donkey, Horse (rarely). 


\subsection{Map of the Study Area}

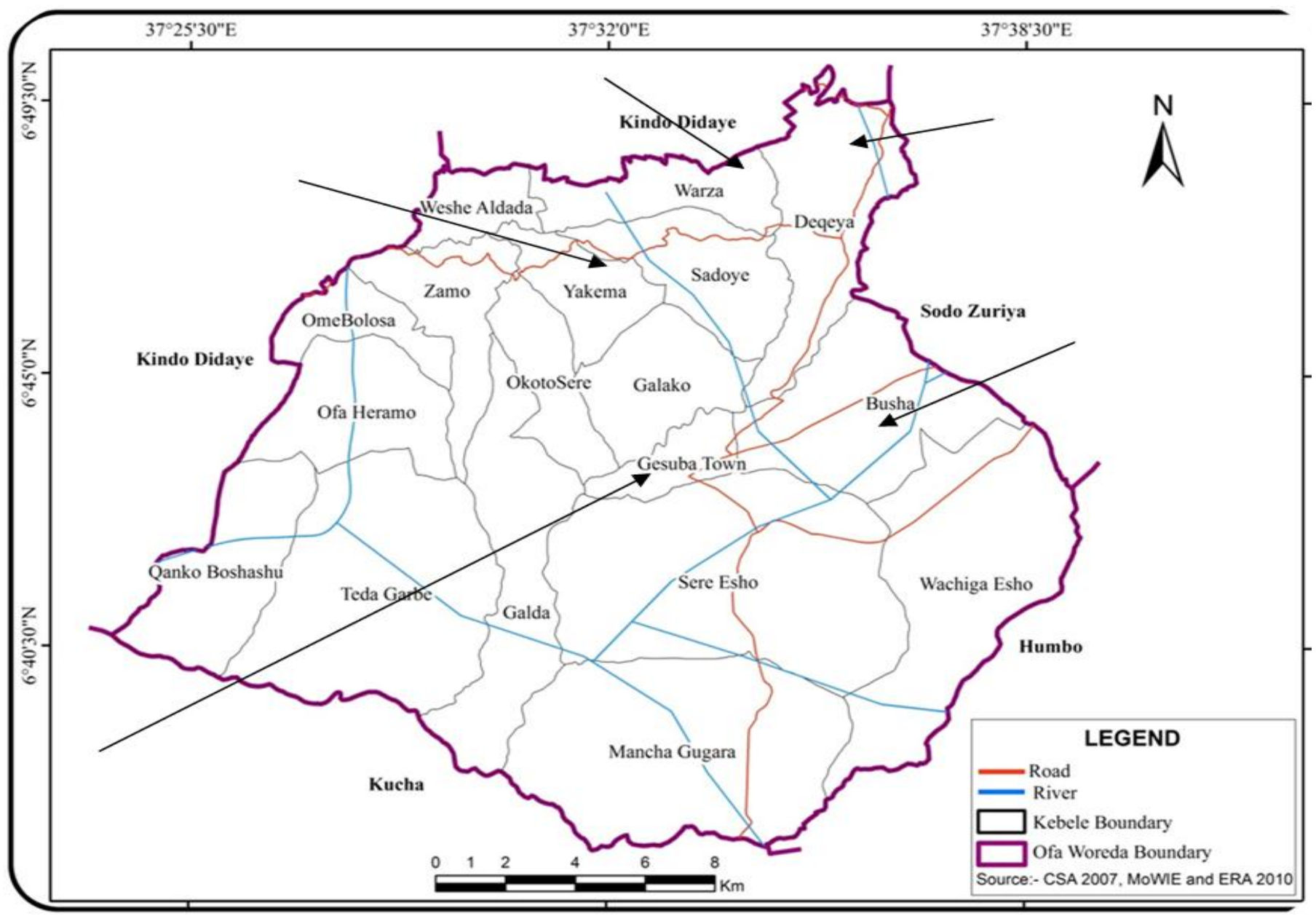

Figure-1. Map of Offa Woreda arrows are indicating the selected sampling kebeles

Source: CSA [21], MoWIE and RRA 2010.

\subsection{Food Groups for Women Dietary Diversity Score (WDDS)}

The food grouping was adopted from FAO nutritional and consumer protection division [27].

\begin{tabular}{l|l}
\hline \multicolumn{2}{c}{ Table-1. Aggregated food groups for WDDS } \\
\hline Food group for WDDS & Food type \\
\hline 1,2 & Starchy staples (roots and tuber, vitamin A rich vegetables and tubers ) \\
\hline 3,6 red palm oil if applicable & Dark green leafy vegetables \\
\hline 5,7 & Other vitamin A rich fruits and vegetable \\
\hline 8 & Other fruits and vegetables \\
\hline 9,11 & Organ meat \\
\hline 10 & Meat and fish \\
\hline 12 & Eggs \\
\hline 13 & Legumes, and seeds \\
\hline Source: FANTA 2009, Developing and Validating Simple Indicators of Dietary diversity
\end{tabular}

\subsection{Description on the Food Group}

The leafy vegetable food is a combination of vitamin A rich vegetables and tubers, dark green leafy vegetables and other vegetables. The fruit group is a combination of vitamin A rich fruits and other vegetables. The meat group is a combination of organ and flesh meat giving sampling codes.

\subsection{Study Design}

A community based cross sectional study design was employed to estimate nutritional status and associated factors of lactating mothers in the study area.

\subsection{Source Population}

Lactating mothers between $15-49$ years of old having children less than 24 months found in study woreda.

\subsubsection{Study Population}

All lactating mothers aged between $15-49$ years and having children less than 24 months and breast feeding during sample collection time in the studied selected six kebeles of Offa woreda.

\subsection{Inclusion and Exclusion Criteria}

\subsubsection{Inclusion Criteria}

Lactating mothers who were breast feeding child of less than 24 months during data collection and resident of the kebeles 


\subsubsection{Exclusion Criteria}

Lactating mothers who were critically sick or unable to stand.

\subsection{Sample Size Determination and Sampling Techniques \\ 2.9.1. Sample Size Determination}

To determine the minimum sample size required for prevalence studies single population proportion formula was used:

$$
N=\frac{Z_{\alpha / 2}^{2} p(1-P)}{d^{2}}
$$

Where,

$\mathrm{N}=$ Sample size

$\mathrm{Z}_{\alpha / 2}=$ Standard normal variable corresponding to the $95 \%$ confidence level $=1.96$

$\mathrm{P}=$ Prevalence of under nutrition among lactating mothers, $50 \%$ estimated

$\mathrm{d}=$ Margin error $($ precision $)=0.05, \mathrm{NRR}=$ Non response rate $(\%)=10 \%$

The total samples size estimated was 422 (four rural and two urban kebeles)

\subsubsection{Sample Selection Methods}

To determine the total required amounts of samples, four rural and two urban kebeles were selected for sampling. The 4 selected rural kebeles were called Busha, Adaye Dakaya, Yakima, and Waraza. While urban Gesuba 01 and 02 Kebeles were considered without randomization due to limited number of population when compared with the rural kebles'. The total sample size divided equally to the studied kebeles (Figure 1).

\subsection{Training and Sampling Procedure}

\subsubsection{Training}

Necessary training was given for data collectors on the topics of household approaching methods and data collection techniques. Up on completion of the trainings, pilot field test was conducted in nearby rural areas of studied kebeles. The purpose of pilot testing is to evaluate how the trainers understood and implement in the field what they were trained on. After pilot test evaluation was made to take corrective action on the error committed during house to house visiting.

\subsection{Sampling Procedure}

Before entering to the sample collection sites, permission from all hierarchy of the administration levels was obtained. After the permission processes completed, randomization was done to select the study kebeles. After the study kebeles were selected again four 'got' from each kebeles were selected in a similar way of randomization method. After the 'gots' were identified, requisition was forwarded to Kebele office to get language translator from Amharic to 'walitigna'. Then, home to home visiting started and the consent form read and translated to local language.

After the targeting groups understood about the objective of the study and decided to participate in the research study, randomization and listing household from selected four 'gots' was started. For each household unique code number was marked on the outer upper parts of the door, which indicates the presence or absence of lactating mother in the coded household. From each randomly selected 'gots' 18 lactating mothers were required; when the availability of lactating mothers in the 'got' exceeds the required level, again randomization method employed. The marked label on each for eligible household was: LW/01, LW/02... and for non eligible, LW/X. Before sampling and interviewing the targeted groups started, first, self introduction to the household was the primary job of the enumerators and coordinator.

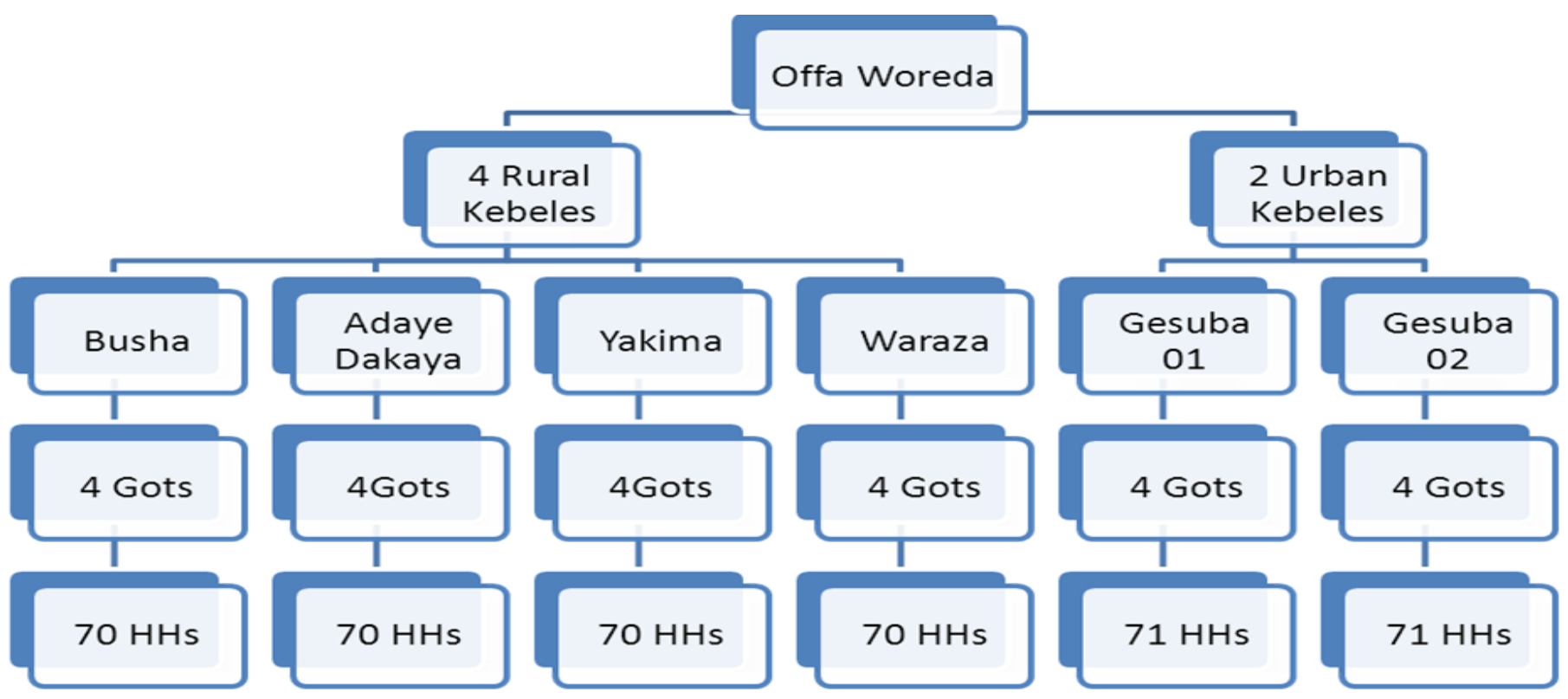

Total HHs (rural kebeles $=70 \times 4=280 \&$ urban kebeles $=71 \times 2=142$ ) $=280+142=422$ Source: Sampling procedure designed by the Author

Figure-2. Schematic sampling procedure in study area of Offa Woreda, 2017 
In each 'got', there was no shortage of lactating mothers and averages of 32 were found and after completing listing, re-randomization was used to work on 18 mothers in each 'got'. Whenever the respondents were not found at home at household visiting time, appointment was taken to re-visit, three times appointment was possible and if not succeeded within the three times appointment, replacement from adjacent got was taken in the same randomization method.

\subsection{Screening for Anemia}

The consent agreement was made between the participants and the data collectors, Blood sample was collected using HaemoCue method according to the guide line manual. The HaemoCue was turned on until 'ready' shown on the screen. Blood sample was drawn from the participants finger after cleaning with alcohol and the finger was held firmly and pricked with disposable lancet. Gentle pressure was applied to extrude a large drop of blood. The finger was wiped off the drop with a dry, lint-free wipe. Wipe away additional one or two large drops, alternately applying and releasing pressure on the finger as needed. The micro cuvette is applied to a drop of blood from the same finger pricked. Blood is drawn into the cuvette by capillary action. Held the cuvette in place until the entire teardrop-shaped cavity is filled with blood. After wiping off any excess blood from the sides of the cuvette, placed in the cuvette holder and inserts it into the HemoCue. Read the hemoglobin [Hb] concentration and record the hemoglobin concentration to one decimal point.

\subsection{Anthropometric Measurements}

Anthropometric measurements were taken using electronics scale balance and height board was used. All data collection was electronically recorded while surveyed in to SUMSUNG Tablet S2 for saving sample entering time.

\subsection{Procedure}

Weights of the lactating mothers was measured to the nearest $0.1 \mathrm{~kg}$ using calibrated portable electronic digital scale with dual display (Seca $874 \mathrm{U}$, Germany) and height was measured to the nearest $0.1 \mathrm{~cm}$ using a portable height-measuring board with a sliding head bar following standard WHO guide line. During height measurements, the eligible lactating mothers were told to remove any hair covering and stand straight on the marked space of the height board. In the same manner, before taking weight measurements, the balance scale was calibrated to normal standard and after all calibration was completed, the respondent advised kindly to remove extra cloths worn and only use light cloths. Lastly, the weight measurement was taken with bare foot. All measurements were done in duplicate to maintain accuracy and whenever the duplicate units divert significantly from each other taking third measurement was compulsory. All data were recorded electronically and for each eligible household GPS was taken to track the exact location of household in case if the needed to be repeated.

\subsection{Study Variables}

\subsubsection{Dependent Variable: Maternal Under Nutrition \\ 2.16. Independent Variables: \\ 2.16.1. Socio-Demographic Factors}

Maternal age, religion, ethnicity, marital status, maternal occupation, maternal educational status, residence and family size and safety, WDDS

\subsection{Maternal Health Care}

Place of delivery, parity, Illness over two weeks prior to the study, anemia, number of Antenatal care (ANC) and Postnatal care (PNC) visit and Water, Sanitation and Hygiene

\subsection{Feeding Practice}

The majority of the respondents used to eat with regardless of cultural norms or other habits any available food at Households. Only very few in number used to eat selectively taking in to consideration the culture norms and habits they learned from their parents.

\subsubsection{Data Quality Control}

The questionnaire was written in English first and translated into the local languages (Amharic). In order to know the age of the child's age, birth certificate obtained from health institute was asked to display on the interview day. In absence of birth certificate some known events, like, holidays, seasons etc, and used to be asked. The sampling tools were pre-tested prior to the data collection training for its accuracy and not encountering problems in the field.

\subsection{Data Processing and Analysis}

\subsubsection{Data Processing}

The collected were analyzed using Stata version 14.2 software in two steps. First, descriptive statistics was used to examine the frequency and distribution of lactating mothers' socio demographic. Secondly, chi square test was applied for association and risk factors for under nutrition of lactating mothers in the study area with the significance value of $\mathrm{p}<0.05$.

\subsubsection{Ethical Consideration}

The ethical approval was obtained from EPHI ethical review committee. The ethical clearance was submitted to the SNNPR, health office and following the zone, woreda and kebeles health offices/centers had received the ethical clearance copies. The lactating mothers were informed about the data collection and its contribution for their future nutritional benefits for the woreda and the country as a whole. The verbal consent was obtained from each participant it was assured that the information obtained from them was maintained confidential. 


\section{Results and Discussion}

\subsection{Results}

\subsubsection{Socio-Demographic Status of the Lactating Mothers}

In this study, a total of 422 lactating mothers aged 15-49 years were participated making a response rate of $\geq$ $99 \%$. The majority of the participants (59.3\%) were in age group of 25-34 and the participants mean age was $25.39 \%$ years. With regarding to ethnicity $99.53 \%$ were wolayita ethnic group. Among the participants, $(87.7 \%)$ were protestant. The majority, $415(98.1 \%)$ of lactating mothers were married. Almost half of the mothers, 217 $(51.3 \%))$ were housewives': concerning educational status of the participants, 207 (48.9\%) was grade one to eight. The percentage of lactating mothers participants were, $111(26.34 \%), 239(56.24 \%)$ and $7(1.65)$ have 1-3, 4-6 and $\geq$ 7 family size, respectively.

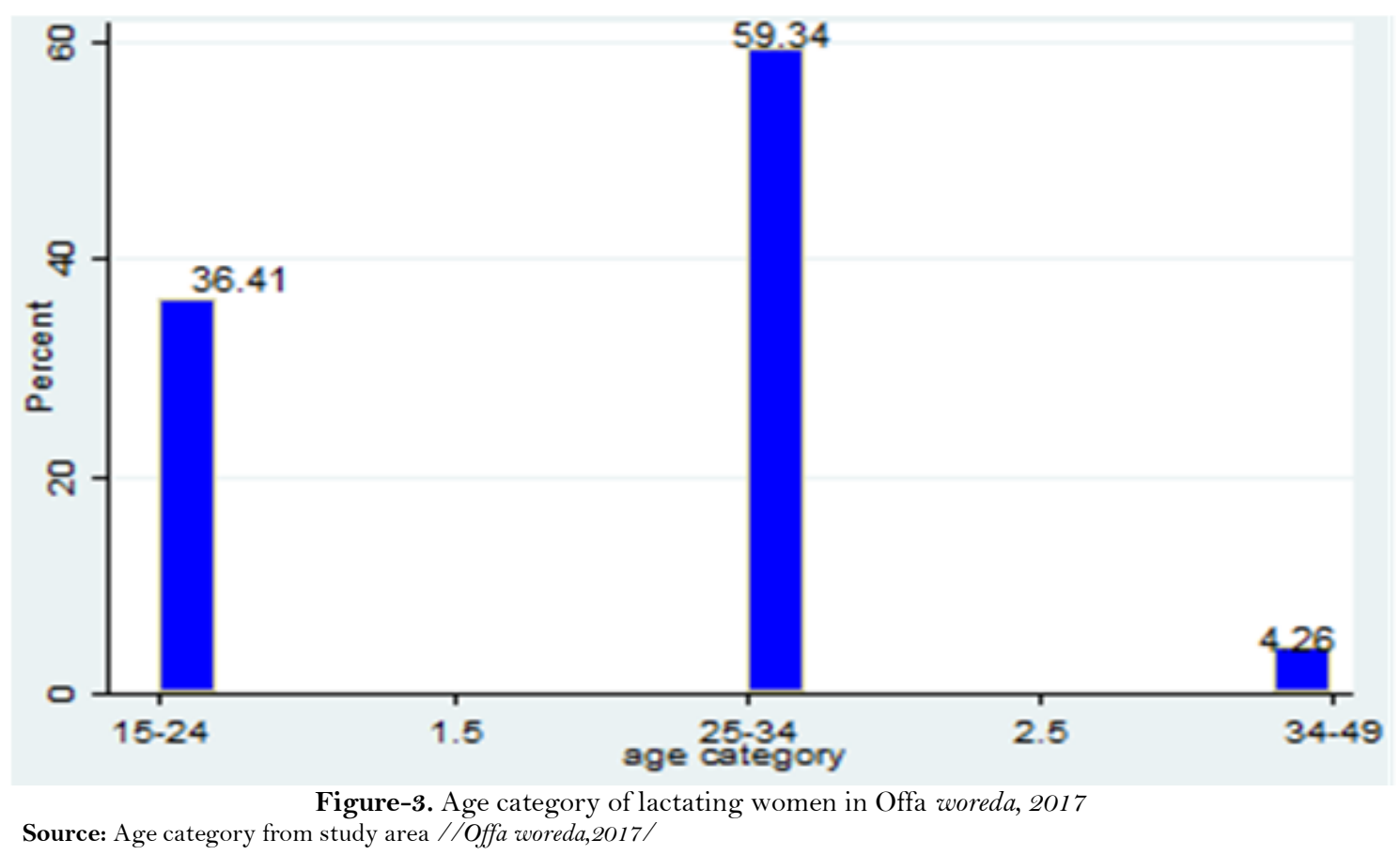

\subsection{Water, Sanitation and Hygiene (WASH)}

Among the lactating mothers, contamination free drinking water and availability of toilet are among the maternal health care services. Accordingly, $90.05 \%$ of lactating women obtained drinking water from pipe line service and the coverage of toilet was $82.23 \%$, with many defects of facility services.

\subsection{Body Mass Indexes of Lactating Mothers}

Among the lactating mothers participants, $15.84 \%\left(<18.5 \mathrm{~kg} / \mathrm{m}^{2}\right), 74.23 \%\left(18.5-24.9 \mathrm{~kg} / \mathrm{m}^{2}\right), 8.27\left(25-29.9 \mathrm{~m}^{2}\right)$ and were $1.64\left(\geq 30 \mathrm{~kg} / \mathrm{m}^{2}\right)$ in their BMI had chronic energy deficiency, normal, over weight and obese respectively as shown in (Figure 5).

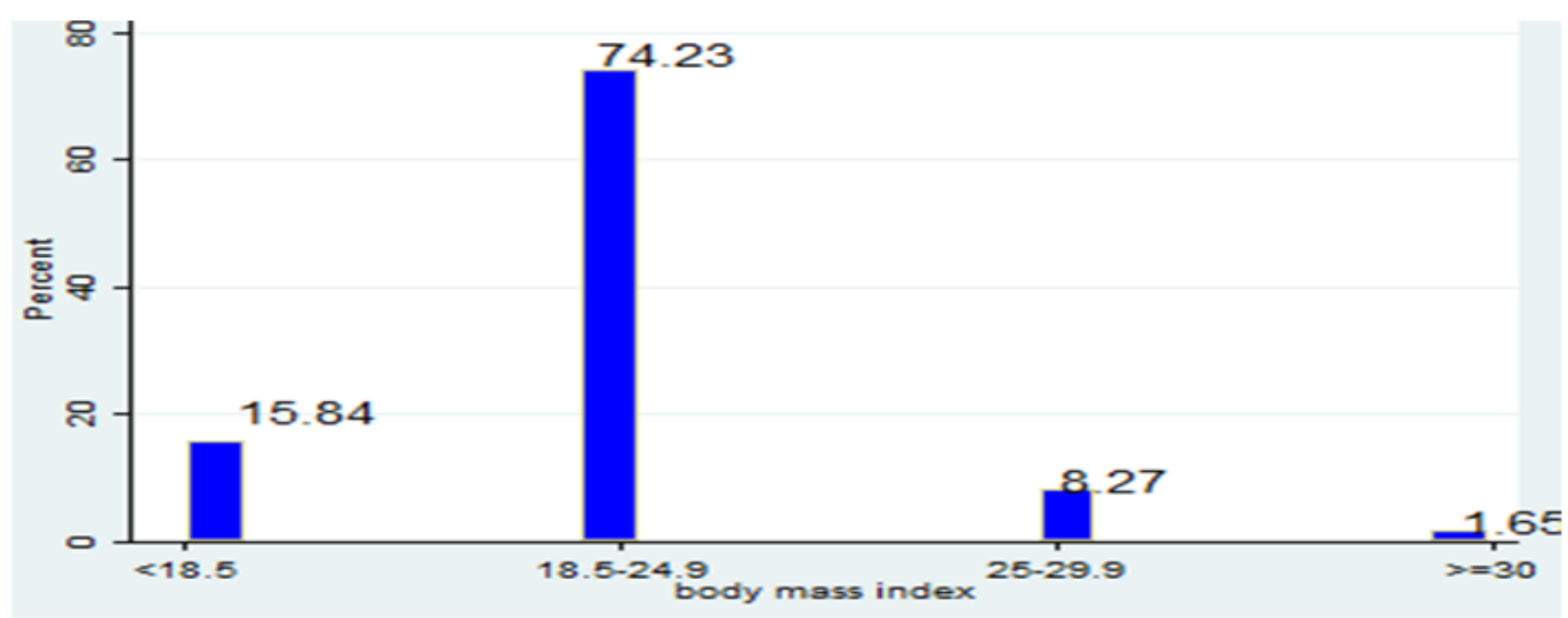

Figure-5. BMI of lactating mothers in the Offa woreda, 2017

Source: From study area of lactating mothers

\subsection{Dietary Diversity of Lactating Mothers (WDDS)}

The recall period of 24 hours was chosen by FAO as it is less subject to recall error, less cumbersome for the respondent and also conforms to the recall time period used in many dietary diversity studies [28]. The analysis of dietary diversity data based on a 24-hour recall period is easier than with longer recall periods. The study conducted in the study area was based on the above guidelines the daily dietary diversity intake per day of lactating mothers was classified as high (>5) $1.2 \%$, medium (4-5) $50.7 \%$ and low $(<3) 47.4 \%$ among the nine food groups recommended for WDDS. 
Table-2. Effect of different risk factors on underweight status of lactating mothers in Offa Woreda, 2017.

\begin{tabular}{|c|c|c|c|c|c|}
\hline Variables & No. observed & Frequency & Percent & $\mathrm{Chi}^{2}$ & P-value \\
\hline \multicolumn{6}{|c|}{ Marital status } \\
\hline Married & 415 & 62 & 14.93 & 19.8820 & 0.001 \\
\hline Others & 8 & 5 & 62 & & \\
\hline \multicolumn{6}{|l|}{ Age } \\
\hline $15-24$ & 154 & 26 & 16.88 & 0.2273 & 0.893 \\
\hline $25-34$ & 251 & 38 & 15.13 & & \\
\hline $35-49$ & 18 & 3 & 16.66 & & \\
\hline \multicolumn{6}{|l|}{ Religion } \\
\hline Orthodox & 47 & 7 & 14.89 & 2.1553 & 0.541 \\
\hline Protestant & 371 & 59 & 15.90 & & \\
\hline Others & 4 & 1 & 25 & & \\
\hline \multicolumn{6}{|c|}{ Educational status } \\
\hline Illiterate & 107 & 16 & 14.95 & 1.3278 & 0.857 \\
\hline $1-8^{\text {th }}$ & 211 & 36 & 17.06 & & \\
\hline $9-12^{\text {th }}$ & 66 & 11 & 16.66 & & \\
\hline Collage & 38 & 4 & 10.52 & & \\
\hline \multicolumn{6}{|l|}{ Family size } \\
\hline $1-3$ & 111 & 20 & 18.01 & 0.6692 & 0.716 \\
\hline $4-6$ & 239 & 37 & 15.48 & & \\
\hline$>7$ & 73 & 10 & 13.69 & & \\
\hline \multicolumn{6}{|l|}{ Occupation } \\
\hline Farmer & 75 & 10 & 13.33 & 6.9564 & 0.044 \\
\hline Student & 10 & 4 & 40.0 & & \\
\hline Merchant & 81 & 11 & 13.58 & & \\
\hline Government & 25 & 3 & 12 & & \\
\hline House wife & 217 & 38 & 17.51 & & \\
\hline Others & 14 & 1 & 7.14 & & \\
\hline Total & & & & & \\
\hline
\end{tabular}

Source: from study area/ woreda, 2017

Table-3. Factors contributing to under nutrition in Offa woreda, 2017

\begin{tabular}{|c|c|c|c|c|c|}
\hline Factor & OR & $\mathbf{Z}$ & p-value & & $95 \%$ CI \\
\hline \multicolumn{6}{|l|}{ ANC Service } \\
\hline$\geq 4$ & 1.27 & 0.98 & 0.326 & 0.7878 & 2.0478 \\
\hline$<4$ & 0.889 & -0.54 & 0.588 & 0.5806 & 1.3608 \\
\hline Family size & 1.11 & 0.067 & 0.084 & 0.9860 & 1.2493 \\
\hline \multicolumn{6}{|l|}{ Education } \\
\hline Able to read and write & 0.168 & 0.17 & 0.869 & -1.8279 & 2.1651 \\
\hline $1-8^{\text {th }}$ & 0.013 & 0.05 & 0.957 & -0.4557 & 0.4817 \\
\hline 9-12th grade & 0.862 & 2.65 & 0.008 & 0.2246 & 1.4993 \\
\hline
\end{tabular}

Source: Data analyzed from study area/Offa woreeda/

Table-4. Effect of various risk factors on nutritional diversity of lactating mothers in the Offa woreda, 2017

\begin{tabular}{l|l|l|l|l|l|l}
\hline & Coef. & Std. Err. & $\mathbf{Z}$ & $\mathbf{P}>|\mathbf{z}|$ & & $\mathbf{9 5 \%}$ CI \\
\hline No ANC & -0.031 & 0.0853 & -0.36 & 0.717 & -0.1981 & 0.1364 \\
\hline Intercept & 0.2333 & 0.3285 & 0.71 & 0.478 & -0.4106 & 0.8772 \\
\hline Education & 0.2374 & 0.0812 & 2.93 & 0.003 & 0.0784 & 0.3965 \\
\hline Intercept & -0.6067 & 0.2487 & -2.44 & 0.015 & -1.0940 & -0.1193 \\
\hline No formal edu. & 1.0186 & 0.17 & 0.869 & -1.83 & -1.8279 & 2.1651 \\
\hline 1-8th Grade & 0.0130 & 0.2391 & 0.05 & 0.957 & -0.4557 & 0.4817 \\
\hline 9-12th Grade & 0.8618 & 0.3253 & 2.65 & 0.008 & 0.2242 & 1.4994 \\
\hline Collage level & 1.0665 & 0.4069 & 2.62 & 0.009 & 0.2899 & 1.8641 \\
\hline Intercept & -0.1686 & 0.1940 & -0.87 & 0.385 & -0.5489 & 0.2117 \\
\hline No formal edu. & 1.0186 & 0.170 & 0.869 & -1.83 & -1.8279 & 2.1651 \\
\hline Occupation & & & & & & \\
\hline Farmer & -0.8804 & 1.2465 & -0.71 & 0.480 & -3.3235 & 1.5628 \\
\hline Merchant & -0.4418 & 1.2453 & -0.35 & 0.723 & -2.8826 & 1.9989 \\
\hline Gov. Employee & 0.6931 & 1.3229 & 0.52 & 0.600 & -1.8996 & 3.2859 \\
\hline Source Data a & & & & &
\end{tabular}

Source: Data analyzed from study area/Offa woreda/

Table-5. Dietary diversity level of lactating mother in the Offa woreda, 2017

\begin{tabular}{l|l|l|l}
\hline \multicolumn{4}{|c}{ Table-5. Dietary diversity level of lactating mother in the Offa woreda, 2017 } \\
\hline High & Daily intake & Freq. & Percent \\
\hline Medium & $(>5)$ & 7 & 1.66 \\
\hline Low & $(4-5)$ & 214 & 50.83 \\
\hline & $(<3)$ & 200 & 47.51 \\
\hline
\end{tabular}

Source: Data analyzed from study area/Offa woreda/ 
Table-6. Dietary diversity status of the Offa woreda, 2017

\begin{tabular}{l|l|l|l|l}
\hline Diversity & \multicolumn{1}{|c|}{ Mean } & \multicolumn{1}{c|}{ Std. dev. } & \multicolumn{1}{c}{ Min. } & Max. \\
\hline Food made from grain & 0.9574 & 0.2023 & 0 & 1 \\
\hline White root and tube & 0.7156 & 0.4564 & 0 & 1 \\
\hline Pulse & 0.4550 & 0.4986 & 0 & 1 \\
\hline Nuts and seeds & 0.0261 & 0.1595 & 0 & 1 \\
\hline Milk and milk product & 0.1754 & 0.3807 & 0 & 1 \\
\hline Organ meat & 0.0024 & 0.0487 & 0 & 1 \\
\hline Meat and poultry & 0.0166 & 0.1279 & 0 & 1 \\
\hline 1 & 0 & 0 & 0 & 0 \\
\hline Egg & 0.0047 & 0.0688 & 0 & 1 \\
\hline Dark green leafy & 0.3649 & 0.4820 & 0 & 1 \\
\hline Vit. A rich vegetable, root and tuber & 0.0308 & 0.1730 & 0 & 1 \\
\hline Other vegetables & 0.7488 & 0.4342 & 0 & 1 \\
\hline Average weight in (Kg) & 52.2580 & 8.9427 & 34.6 & 108.2 \\
\hline Average height (cm) & 157.2863 & 6.1155 & 138.1 & 179.2 \\
\hline Source: Data analyzed from study area/Offa woreda/ & & & &
\end{tabular}

\subsection{Maternal Health Care}

Among the lactating mothers, (79.86\%) of them ANC service $\geq 4$ times, (78.44\%) Iron tablet, (23.78\%) vitamin A obtained during the last birth of pregnancy and (52.61\%) have given birth at health institute during their last child. The blood hemoglobin test in the study woreda shows $11.37 \%$ among the participants were the hemoglobin level in the blood was $\leq 12 \mathrm{~g} / \mathrm{dl}$ (Table 7 ).

Table-7. The effect of anemia on dietary diversity of lactating mother in Offa woreda, 2017

\begin{tabular}{l|c|c|c}
\hline Anemia status nutritional diversity & Anemic & Normal & Total \\
\hline DDS $\geq 4$ & $26(11.98 \%)$ & $196(88.02 \%)$ & 217 \\
\hline DDS $<4$ & $22(10.78 \%)$ & $182(89.22 \%)$ & 204 \\
\hline Source: Lactating Mothers from Offa woreda 2017
\end{tabular}

Source: Lactating Mothers from Offa woreda, 2017

Hemoglobin $<12 \mathrm{~g} / \mathrm{dl}$ is anemic and $\geq 12 \mathrm{~g} / \mathrm{dl}$ normal (WHO guide line).

The minimum and maximum recorded values of hemoglobin were 9.3 and $17.2 \mathrm{~g} / \mathrm{dl}$, respectively. The recorded mean value was $13.56 \pm 1.35 \mathrm{~g} / \mathrm{dl}$. The effect of being normal or anemic had no significant ( $\mathrm{p}>0.05)$ effects on dietary diversity intake of lactating mothers. The minimal amount of animal source foods intake in their daily meal could likely be the reason why it was not observed as significant effect.

\section{Discussion}

The study conducted on the particular community members of lactating women in SNNPR, Wolayita zone Offa Woreda in the selected six kebeles showed that among the participants, $15.84 \%$ with $95 \%$ CI $(12.52,19.72)$ were $<18.5 \mathrm{~kg} / \mathrm{m}^{2}$ in their BMI. This result is comparable with chronic energy deficient of lactating women in Pakistan $15.1 \%$ [18]. The nutritional status of the study area was much less than the national level $(27 \%)$ as reported by Food and Agriculture Organization [3]. The chronic energy deficiency status identified in the study area (Offa woreda) was much difference from chronic energy deficiency in Babile woreda $41.7 \%$ and $54.4 \%$ during post and pre-harvesting, respectively [29]. The chronic energy deficiency in other parts of the developing countries showed, in Bangladaesh (30\%) UNICEF/ Hossain, et al. [30] and Vietnam (28.3\%) [31]. The study conducted at Ambo district of lactating mothers was 21.5\% [29] which was higher than the result obtained in this study.

When the blood hemoglobin in the study woreda was checked out, the prevalence of anemia in the study woreda was $11.4 \%$. This indicates that among the participants $11.4 \%$ had $<12 \mathrm{~g} / \mathrm{dl}$ hemoglobin in their blood. No severe case of anemia was observed for lactating mothers in the studied area (Table 7).

In EDHS 2016 report, the prevalence of anemia in all regions and community members was assessed, from the report. The studied area observation was somehow compared with the anemia prevalence of Addis Ababa (15.9\%) and Amhara (16.3\%) mothers by EDHS 2016.however the national prevalence of anemia was $16.4 \%$ and $24.4 \%$ urban and rural, respectively as it was reported by EDHS (Ethiopia Demographic and Health Survey) [6].

According to Kennedy and Nantel [14] project survey, nine types of dietary diversity of food groups were recommended for reproductive age women; out of the nine, at least four types of Dietary foods are needed in their daily meal at minimum level. The daily, dietary intake of lactating mothers in the studied woreda was classified in to high level $(>5)$, medium or optimum (4-5) and minimum $(<3)$ per day in their daily meal. The result obtained in the study area was, $(>5)$ 1.2\%, medium or optimum) (4-5) $50.7 \%$ and low $(<3) 47.4 \%)$. From the result, the minimum and medium are comparable; almost half of the lactating mothers used to get minimum level of dietary food in their daily meal. The two major staple foods in the study woreda were food made up of grains (95.7\%) and wheat and root crops $(71.7 \%)$. However, the rests of dietary diversity were much below recommended level for lactating mothers.

Contamination free and treated water is one of the health factors for all human beings. In the study woreda, only $52.2 \%$ of the participants were getting drinking water from the pipeline around their residences which are slightly less than the reported percentage value of the EDHS (Ethiopia Demographic and Health Survey) [6] $56.6 \%$ at rural level. However, with respect to hygienic condition, only $18.7 \%$ had a habit of washing their hands before taking food. Among the lactating mothers only $15.2 \%$ had knowledge about the cause of disease like diarrhea. Most of the lactating mothers $(80.2 \%)$ were not counseled on nutritional food when they are visited for other health care services and only 19.8\% were counseled on nutritional issues. Thus shows the knowledge level of nutritional food was very limited. 
The socio-demographic of the lactating mothers in the studied woreda showed that percentage of the participants 56.5 and 26.2 households had 4-6 and 1-3 family size, respectively. The education status of the participants was also identified and the interview made with them $25.4 \%$ of the participants, had no formal education which had less than the reported value $(47.8 \%)$ [6]. The minimum obtained participants percentage education level were high school and above. Among the participants 98.8 were married. 59.3\% were in the age group of 25-34 and 51.4\% were housewife. The number of children in each study participants was identified and among the participants 68.9 and $37.8 \%$ had 1-3 and 4-6 children, respectively. With regarding to the ethnicity $99.9 \%$ were walayita; the majority or $415(98.1 \%)$ lactating mothers were married. With regarding to maternal health care, $35.9 \%$ participants had obtained ANC service three times followed by $29.4 \%$ who got four times. The rest $(34.7 \%)$ of the participants were less when compared with the two service periods.

The risk factors for chronic energy deficiency in this study were marital status and education level with ( $\mathrm{p}<$ 0.05). While the rest were not risk factors for chronic energy deficiency (Table 1).

In this studied area, age category, religion, Dietary Diversity and health care services like ANC, didn't have significant effects on the nutritional status of the lactating mothers. This was similar to the study conducted at Ambo, rural areas dietary diversity had no significant $(\mathrm{p}>0.05)$ effects on BMI of lactating mothers [32]. In this studied woreda, the non-educated mothers were more likely to be malnourished than those High school and college levels as indicated in (Table 2)

\section{Conclusion}

The results obtained from this study showed that chronic energy deficiency status of the lactating women in the study woreda was much lower than the chronic energy deficiency reported at national and regional levels. The daily dietary diversity intake of low and medium level were comparable, indicating insufficient level for lactating mothers to maintain themselves health and the child breast feeding from this portion. The percentages of lactating mothers who have reached high school and above level were very low; thus education had significant risk factors on chronic energy deficiency of lactating mothers. In order to improve the knowledge level of lactating mothers, continuous education is required. Besides, the dietary diversity of lactating mothers should be improved by adopting multi cropping system in the studied Offa woreda.

\section{References}

[1] R. Black, C. Victora, and S. Walker, "Maternal and child under nutrition and overweight in low-income and middle income countries," Lancet, vol. 382, pp. 15-39, 2013.

[2] H. Kiday, M. Afework, and G. Meron, "Feeding practice s, nutritional status and associated factors of lactating women in samre woreda, South Eastern zone of Tigray, Ethiopia," Nutrition Journal, vol. 12, pp. 1-11, 2013.

[3] Food and Agriculture Organization, The state of food and agriculture 2010-2011 women in agriculture; closing the gender gap for
development. Rome: FAO, 2011 . development. Rome: FAO, 2011.
CSA, Population and housing census report at national level. Addis Abeba, Ethiopia: Central Statistical Agency, 2015.

[4] CSA, Population and housing census report at national level. Addis Abeba, Ethiopia: Central Statistical Agency, 2015. USA," 2011.

[6] EDHS (Ethiopia Demographic and Health Survey), "Central Statistical Agency of Ethiopia, Addis Ababa, Ethiopia, The DHS Program ICF, Rockville, Maryland, USA," 2016.

[7] D. H. Temesgen, F. G. Habtamu, W. Dessalegn, and N. K. Dunkana, "Nutritional status and associated factors among lactating mothers in Nekemte Referral hospital and health centers, Ethiopia," Food Science and Quality Management, vol. 35, pp. 64-72, 2015. View at Google Scholar

[8] Z. Eshetu, "Household food insecurity and factors associated with undernutrition among lactating mothers in rural Ambo District, West Shewa Zone, Oromia Region, Ethiopia," p. 12, 2016.

[9] WFP, "Special Report on Crop and Food Security Assessment Mission to Ethiopia: Integrating the Crop and Food," 2010.
[10] EHNRI (Ethiopian Health and Nutrition Institute), "Micro nutrient survey, the role of education in nutrition. Addis Ababa, Ethiopia," 2010.

[11] R. Jayawardena, N. Byrne, J. Soares, P. Katulanda, B. Yadav, and P. Hills, "High dietary diversity is associated with obesity in Sri Lankan adults: An evaluation of three dietary scores," BMC Public Health, vol. 13, p. 314, 2013. View at Google Scholar $\mid$ View at Publisher

[12] WHO, "Improving nutrition outcomes with better water, sanitation and practical solutions for policies and programme. Hygiene problems in developing countries," 2015.

[13] M. Savy, Martin-Prével, Y. Traissac, P. Eymard-Duvernay, and S. Delpeuch, "Dietary diversity scores and nutritional status of women change during the seasonal food shortage in rural Burkina Faso," 2006.

[14] G. Kennedy and G. Nantel, "Basic guidelines for validating dietary diversity score as an indicator of adequate micronutrient intake." Retrieved http:// www.ftp.fao.org/ag/agn/nutrition/ddsvalidation.pdf. [Accessed June, 2017$], 2006$.

[15] FANTA (Food and Nutrition Technical Assistance-United States Agency International Development), "Overview of the nutrition situation in the seven countries in Southeast Asia. Retrieved http://www.fantaproject.org/sites/default/files/download/Southeast-Asia-Nutrition-Overview-Apr2014.pdf." [Accessed March $21,2017], 2014$ [16] Food and Agriculture Organization, "FAO statistical yearbook 2012."
http://www.fao.org/docrep/015/i2490e/i2490e03a.pdf. [Accessed September 2016], 2012.

[17] R. Bain, R. Cronk, J. Wright, H. Yang, and T. Slaymaker, "Fecal contamination of drinking water in low- and middle-income countries," 2014.

[18] A. Zulfiqar, B. Lamberti, and J. Haider, "Diarrhea morbidity, the challenge of malnutrition among women and children globally," 2011.

[19] V. Brinda, D. Getsie, V. Swarna, and R. Bhavani, "Dietary diversity and women's BMI among farm households in rural India," 2015.

[20] D. P. Saskia, H. Brinkman, P. Webb, S. Godfrey, I. Darnton-Hill, H. Alderman, D. Richard, S. Ellen, P. Martin, and W. Bloem, "How to ensure nutrition security in the global economic crisis to protect and enhance development of young children and our common future," Journal of Nutrition, vol. 140, pp. 138S-142S, 2010. View at Google Scholar | View at Publisher

[21] CSA, Population and housing census report at national level. Addis Abeba, Ethiopia: Central Statistical Agency, 2006.

[22] A. Ahmed, R. Hill, L. Smith, D. Wiesmann, and T. Frankenberger, The world's most deprived: Characteristics and causes of extreme poverty and hunger: Intl Food Policy Res Inst, 2007.

[23] Food and Agriculture Organization International Fund for Agricultural Development World Food Program, The state of food insecurity in the world 2015. Strengthening the enabling environment for food security and nutrition. Rome: FAO, 2015.

[24] R. Nigatu, A. Gordon, L. Zello, E. Getahun, J. Carol, and L. Henry, "Food insecurity and hunger," 2011.

[25] B. Almaz, "Determinants of rural household food in security in Wolaita Zone, Ethiopia," 2016.

[26] W. Worku, F. Mazengiya, and M. Kifle, "Knowledge, perception and utilization of postnatal care of mothers in Gondar 'zuria' District, Ethiopia," 2014. 
[27] FANTA, "Dietary diversity as a measure of the micronutrient adequacy of women's diets: Results from Rural Mozambique Site," 2006.

[28] M. Ruel, "Is dietary diversity an indicator of food security or dietary quality? A review of measurement issues and research needs," Food Nutrition Bulletin, vol. 24, pp. 231-232, 2003. View at Google Scholar | View at Publisher

[29] A. Kader, "Increasing food availability by reducing postharvest losses of fresh produce," Acta Hort, vol. 682, pp. 2169-78, 2005. View at Google Scholar | View at Publisher

[30] M. Hossain, P. Bharati, S. Aik, P. Lestrel, A. Abeer, and T. Kamarul, "Body mass index of married Bangladeshi women: Trends and association with socio-demographic factors," Journal of Biosocial Science, vol. 44, pp. 385 - 399, 2012. View at Google Scholar $\mid$ View at Publisher

[31] N. M. Nkamori, N. Ninh, H. Isomura, N. Yoshiike, V. Hien, B. Nhug, N. Nhien, T. Nakano, N. Khan, and S. Yamamoto, "Nutritional status of lactating mothers and their breast milk concentration of iron, zinc and copper in rural Vietnam," Journal of Nutrition Science Vitaminol, vol. 55, pp. 338-345, 2009. View at Google Scholar $\mid$ View at Publisher

[32] Z. Eshetu, "Household food in security and factors associated the under nutrition among lactating mothers in Rural Ambo, West Shewa," 2016. 